\title{
Using GIS to derive spatial indicators of pedestrian exposure to urban traffic noise
}

\author{
Paulo Rui Anciães and Giles Atkinson \\ London School of Economics and Political Science, United Kingdom
}

Cite as: Anciaes, P., Atkinson, G. (2012) Using GIS to derive spatial indicators of pedestrian exposure to urban traffic noise., in S Rauch, G Morrison G, S Norra, N Schleicher (Eds.) Urban Environment. Springer, Dordrecht., pp.323-332.

\begin{abstract}
Pedestrians are particularly susceptible to urban environmental nuisances such as transport noise. While we have methods for measuring pedestrian noise exposures in specific locations, policy-makers also require larger-scale evidence on how their policies affect exposures. This paper assesses the role of pedestrian mobility on exposures of people living and working in different areas and using different travel modes at different times. Two GIS-based indicators measure exposure. Non-employed individuals are exposed along the routes to nearby locations and employed individuals are exposed on the walking sections of the optimal routes to work.

The indicators are applied in the assessment of the expansion of the road network and traffic in the Lisbon Metropolitan Area. The exposures of the non-employed have increased in central areas, affecting elderly residents. Areas above exposure standards tend to have employed populations with above-average qualifications while areas below standards have below-average qualifications. Areas above standards for both employed and non-employed individuals also have below-average qualifications.
\end{abstract}

\section{Introduction}

Policy-makers are increasingly interested in promoting walking as an environmentallyfriendly type of mobility. However, the quality of this mobility also depends on environmental factors. Transport noise has a particularly marked effect on pedestrians, due to its effects on psychological wellbeing, safety and social interaction $[1,2]$. While researchers have developed methods for measuring exposures in specific locations and inside different types of vehicles, there is less evidence on how transport and urban policies affect pedestrian exposures and create conflicts between motorised and pedestrian mobility throughout the city. These conflicts may be politically relevant, if they are to the disadvantage of groups vulnerable to losses in pedestrian mobility or 
with higher reliance on walking, such as the elderly or the low-income populations [3]. This disadvantage may also have a geographical dimension, if the areas where these groups walk have higher noise levels.

The objective of this paper is to map indicators of pedestrian noise exposure that integrate the patterns of daily mobility of the population in each neighbourhood, including the destinations accessed at different times of day and the travel modes used to access them. The hypothesis is that individuals are most vulnerable to noise when walking around their neighbourhood or on their way to work, including the walking sections of trips by private or public transport. The paper adds to studies that quantify and map pedestrian mobility, which have been mostly confined to aspects related with urban planning, such as pedestrian accessibility and street "walkability" [4,5], and seldom analysed the extent to which walking is restricted by motorised traffic. The work also adds to studies on the distribution of noise according to age and socioeconomic status [6]. This literature usually assumes that exposure occurs at home and is the same for all individuals in the unit of analysis. The distributions are then based only on residence location, not taking into account that individuals move across different parts of the city throughout the day. The contribution of our work is to consider choices over destinations and travel modes as additional factors explaining the distribution of exposures.

The method is applied to the case of the Metropolitan Area of Lisbon, analysing the effects of the expansion of the motorway network and private transport usage and urban fragmentation in the period between the last two population censuses. The assessment of the quality of pedestrian mobility is relevant in face of trends common with other European cities such as population ageing, macroeconomic instability and cultural shifts towards healthier lifestyles, which have increased the relevance of walking and the need to take into account the needs of vulnerable groups.

\section{Methods}

The analysis is conducted at the level of the census enumeration district. However, to take into account internal variations in land use, the indicators of noise exposure are assessed for a series of points representing all different contiguous areas of residential land inside each district, and then averaged for the district according to the estimated spatial distribution of the population. The calculations use GIS network models for the private, public and pedestrian transport systems in 1991 and 2001. Exposures depend on the distribution of noise levels throughout the day. The analysis is restricted to the period 6:00-00:30. All the variables used to estimate exposures are then split into two periods: peak (6:30-9:30 and 16:30-19:30) and off-peak (9:30-16:30 and 19:30-0:30).

Peak and off-peak noise surfaces are modelled for 1991 and 2001. In a first stage, noise levels are calculated for each segment of the road and rail networks, based on the segment characteristics and on estimated traffic levels, composition and speeds. These variables are obtained by assigning commuting and non-commuting private transport 
flows to the road network and by modelling bus and train routes and schedules. Network speeds are based on the link characteristics and congestion. In a second stage, the noise levels on the network are used to obtain levels in a $40 \mathrm{~m}$ grid covering the study area, considering the noise propagation over space and the geometric relationships between each point and the noise source. This estimation uses several GIS datasets representing the local natural and built environment. Finally, the noise level at each point of the grid combines the modelled noise from the road and rail networks and noise from other sources such as major industries, airports and flight paths. Noise from these sources is obtained by extrapolating data available for recent years. Noise levels at railway and underground stations are modelled separately, using formulas from the literature. The grid is finally converted to a surface, giving the peak and off-peak equivalent continuous sound level in every location of the metropolitan area.

We consider that the mobility of the residents in each district depends on their employment condition. Different assumptions apply for the destinations, travel modes and pedestrian noise exposures of the non-employed and employed population [Table $1]$.

Table 1: Exposure to transport noise of a neighbourhood's population

\begin{tabular}{|c|c|c|c|}
\hline $\begin{array}{c}\text { Employment } \\
\text { status }\end{array}$ & Destinations & Transport mode & Exposure \\
\hline Not employed & Nearby places & Walk & \multirow{2}{*}{ Walking to destination } \\
\hline \multirow{4}{*}{ Employed } & \multirow{4}{*}{ Workplace } & Walk & \\
\hline & & \multirow[t]{2}{*}{ Public transport } & $\begin{array}{l}\text { Walking to/from/between stations/bus } \\
\text { stops }\end{array}$ \\
\hline & & & Waiting at stations/bus stops \\
\hline & & Private transport & Walking to/from car parks \\
\hline
\end{tabular}

\section{Non-employed population}

For the non-employed population, pedestrian mobility is a means for intra or interneighbourhood accessibility. A set of possible destinations is built for every district in 1991 and 2001. This set is obtained by a sampling of all inhabited 40m cells in a grid covering the study area and is the largest possible set such that all points are at least $400 \mathrm{~m}$ apart and the probability that each point is included in the set is proportional to its population density. It is assumed that pedestrians only consider destinations within $800 \mathrm{~m}$ straight-line distance. These restrictions ensure that each district has a maximum of 12 possible destinations. Each destination is assigned an attractiveness score specific to each origin and depending on the number of opportunities for social interaction. This number is measured by the population living nearby, which is estimated by assigning the population of the metropolitan area to its nearest point in the set of all pedestrian destinations. For destinations at 500-800m straight-line distance, this number is multiplied by $50 \%$ to correct for the effect of distance on 
propensity to walk. Individuals are then exposed along the optimal walking routes from the representative points of the district to the destinations of that district. Optimality is based on walking time, with speeds depending on slopes.

The indicator of pedestrian noise exposure of the non-employed population in district $i\left(E n_{i}\right)$ is the sum for all destinations $k$ of the length-averaged noise levels on the optimal routes to each destination multiplied by the probability that individuals choose that destination. This probability is the proportion of that destination's attractiveness for the population in that district $\left(P_{i, k}\right)$ and the attractiveness of all the destinations $\left(\sum_{k} P_{i k}\right)$. We assume the number of pedestrian trips is constant throughout the day and as such the noise levels $N_{i, k}$ are a weighted average of peak and off-peak levels, where the weights are the number of hours in each period.

$$
E n_{i}=\sum_{k} \frac{P_{i, k}}{\sum_{k} P_{i, k}} * N_{i, k}
$$

\section{Employed population}

For the employed population, walking is either a means to complement the trip to work by motorized transport or to access directly the workplace. Motorized transport users access a set of destinations representing major centres of employment, including 207 and 240 points in 1991 and 2001. This set was constructed considering sectoral employment and business data at the municipality and sub-municipality level. Land use maps and other ancillary information were then used to identify precise locations for the employment in each sector. Workers walking to work access the same set of destinations defined above for the non-employed population. However, the attractiveness score of each destination is an average of the attractiveness for workers of different sectors, with local population measuring attractiveness for the service sector and agricultural and industrial space measuring attractiveness for the corresponding sectors.

Individuals are exposed on the walking sections of the optimal routes to work. Private transport users walk between car parking areas and the workplace. Parking is only modelled for origins and destinations in major cities, assuming that individuals have access to free parking close to their residence and workplace in the other cases. Public transport users walk between home/work and bus stops/train stations and during interchange to different modes or services. We assume that individuals are also exposed to transport noise during waiting time at all stops, to account for the fact that the usual noise loudness at bus stops tends to exceed standards in our study area, due to the location of many of them along major transport arteries. For both motorized modes, optimal routes depend only on time, including the effect of congestion. Public transport times also include waiting and interchange time and penalties for delays and variations in service headway due to congestion. 
The daily exposure to noise of users of motorized mode $m$ (private or public transport) starting work during period $p$ and ending at the corresponding period $p$ ' is given by the equivalent exposure level of the exposures in all walking sections of the return trip to work. In the formula below, $T_{i, j, m, p}$ and $T_{j, i, m, p^{\prime}}$ are the total walking times in the trip from district $i$ to destination $j$ using mode $m$ during periods $p$ and $p$ '

respectively. $N_{i, j, m, p}$ and $N_{j, i, m, p^{\prime}}$ are the length-averaged noise levels in the walking routes used, or the noise level at the stop or station, in the case of waiting time. In the cases where car parking is not modelled, we assume that exposure is the background noise level.

$$
E_{i, j, m, p}=10 * \log _{10}\left[T_{i, j, m, p} * 10^{\frac{N_{i, j, m, p}}{10}}+T_{j, i, m, p^{\prime}} * 10^{\frac{N_{j, i, m, p^{\prime}}}{10}}\right]
$$

The indicator of pedestrian exposure of the employed population in district $i$ is the weighted average of the exposures of workers travelling to each destination at each period of the day by each transport mode. In the formula below, $F_{i, j, m, p}$ is the proportion of all workers in district $i$ that start work during period $p$ at location $j$ and travel by motorized mode $m$ and $W_{i, k, p}$ is the proportions of workers starting work during the same period $p$ at location $k$ accessed by walking. $E_{i, j, m, p}$ is the exposure in the pedestrian sections of the journey to work by motorised mode, as defined above, while $E_{i, k, p}$ is the length-weighted average of noise levels in the routes taken by workers walking to work. The disaggregation of destinations, times of day and travel modes at each district uses several datasets from the population census and mobility surveys.

$$
E e_{i}=\sum_{j, m, p} F_{i, j, m, p} * E_{i, j, m, p}+\sum_{k, p} W_{i, k, p} * E_{i, k, p}
$$

\section{Application}

The approach is used to map the distribution of pedestrian noise exposures in the Lisbon Metropolitan Area in 1991 and 2001. Figures 1 and 2 show the exposures for the non-employed and employed population. In both cases, the values are generally higher and less variable than those usually found in literature mapping exposures at home. This is because pedestrians visit with the highest probability the noisiest areas surrounding their residence, that is, the most populated areas (in the case of the nonemployed) and the areas where bus stops and train stations are location (in the case of the employed population). 


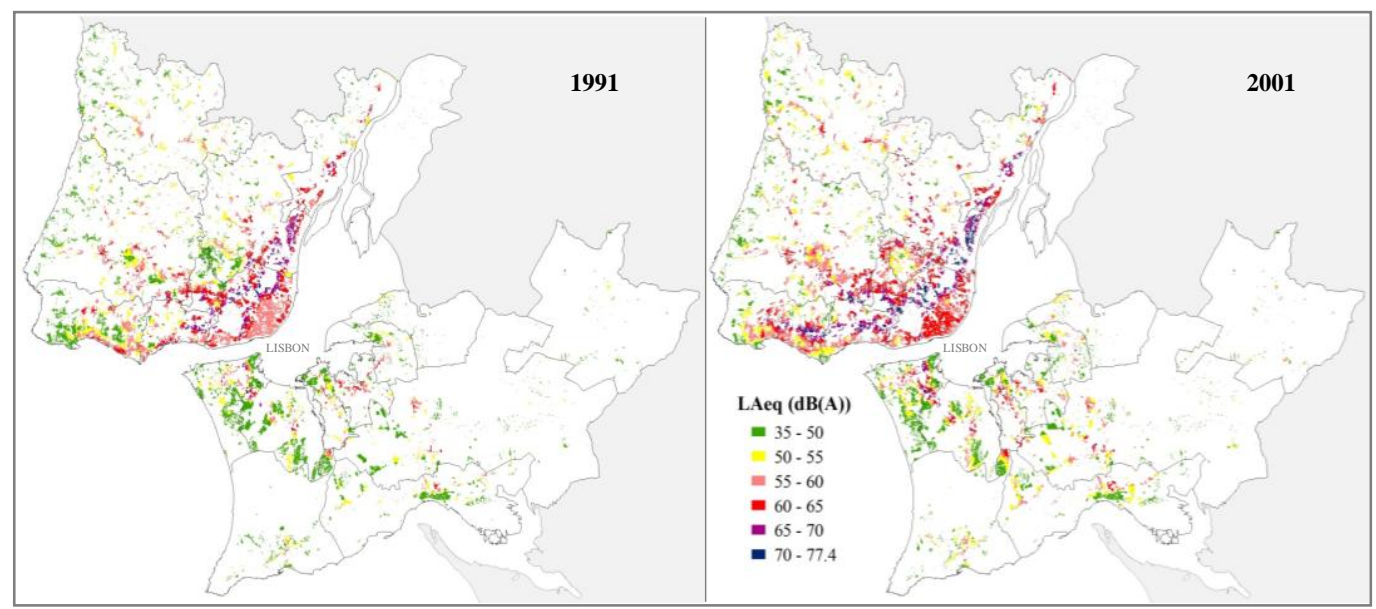

Fig.1: Pedestrian noise exposures of the non-employed population

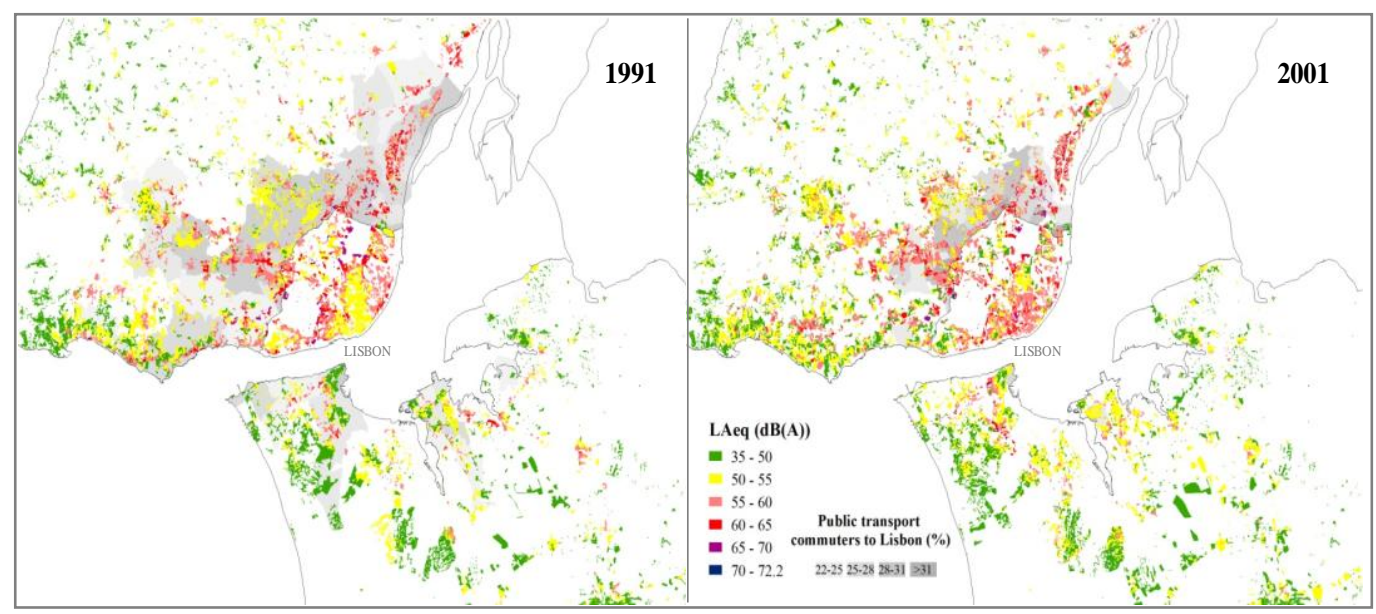

Fig.2: Pedestrian noise exposures of the employed population

The exposures of the non-employed population are high (55-65 $\mathrm{db}(\mathrm{A}))$ in main centre of the metropolitan area (Lisbon) and very high (>65 $\mathrm{db}(\mathrm{A}))$ in the surrounding suburban areas. Values are also high alongside parts, but not all, of the main transport corridors to Lisbon. The affected areas in these corridors tend to be those that are surrounded by several motorways, where most pedestrian destinations have high noise levels. The situation is especially acute in the Northeast corridor, which is formed by a long and narrow strip of residential land sided by national-level road and rail infrastructure, along flight paths and beside the major industrial corridor in the metropolitan area. There is also a high degree of variability of exposures within each municipality, which is partly independent of the location of the major transport infrastructure. From 1991 to 2001, exposures have increased in most districts, with values above standards spreading to the semi-rural areas of the outer municipalities. The densification of the motorway network around Lisbon also led to the increase of exposures in areas where they were already high. Exposures are also above average in some of the new urban developments in less dense areas. As growth has been largely 
discontinuous, these areas often have few pedestrian destinations, accessible only by using busy roads.

The exposures of the employed population are generally lower than those of the non-employed population and follows pattern mainly based on levels of public transport commuting to Lisbon. This is explained by the fact that destinations in Lisbon tend to have the highest noise levels and as such, waiting at stations and bus stops on the return trip will contribute to higher daily exposures. Exposures above standards tend to be higher in the Northwest and Northeast corridors. Despite having comparable proportion of the workers commuting to Lisbon by all transport modes, the West corridor shows lower exposures than those two corridors, due to the smaller proportions of public transport users, as these users are generally more exposed to noise. However, despite the overall decrease in the proportion of public transport commuters to Lisbon, exposures did not decrease in any of the corridors from 1991 to 2001. This may be explained by the increase of noise levels in Lisbon, which is reflected in the noise exposures of the population working in this city.

These distributions are then analysed in terms of the underlying socio-economic profile of each district. The analysis focuses on two main vectors of variables, obtained by a previous factor analysis to census data. For each indicator, we focus on one vector, chosen to take into account the vulnerable groups identified in the introduction. In the case of the non-employed population we focus on a vector labelled Age, associated with the age of both population and buildings. In the case of the employed population we focus on a vector of Qualifications, associated with variables measuring educational and professional qualifications and with other variables linked to socio-economic status, such as large and large and owner-occupied dwellings, values of rents and mortgage payments. Both vectors are standard variables, with zero mean and unit variance.

Fig. 3 shows the population-weighted averages of these factors for each class of noise exposure. The top part of the figure shows that in the period concerned there was a structural change in the distribution among age groups of exposures for the nonemployed population. In fact, apart from the most extreme exposure levels, which remain associated with negative values of the Age variable, the average value of the variable for noise exposures above $55 \mathrm{~dB}(\mathrm{~A})$ has increased substantially, while the averages for less noisy values (45-55 db(A)) shifted from positive to negative. Fig.1 suggests that these changes may be linked to the increase of noise levels in the city of Lisbon and other central areas, as the population in these areas is considerably older than in the rest of the metropolitan area.

The distribution of exposures for the employed population according to qualification levels (bottom part of Fig.3) has remained unchanged from 1991 to 2001, showing an almost perfect separation between above-average qualifications in areas above standards and below-average qualifications in areas below standards. The exception to this pattern occurs in the areas with the smallest noise exposures, which correspond to isolated areas in rural municipalities [Fig.2]. These regions tend to have populations with relatively low qualification levels. 


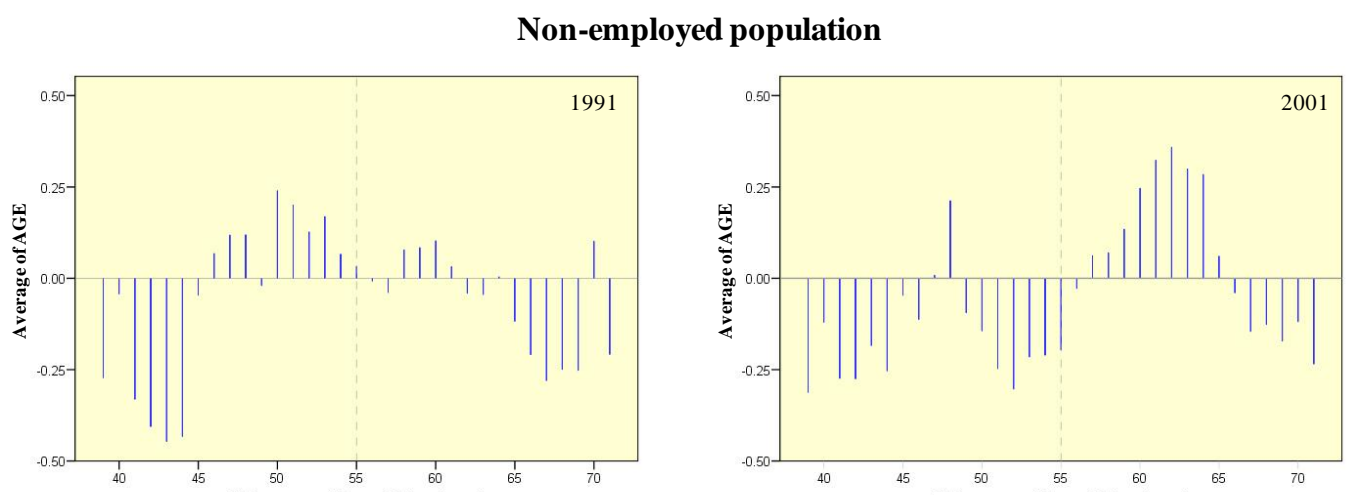

Employed population
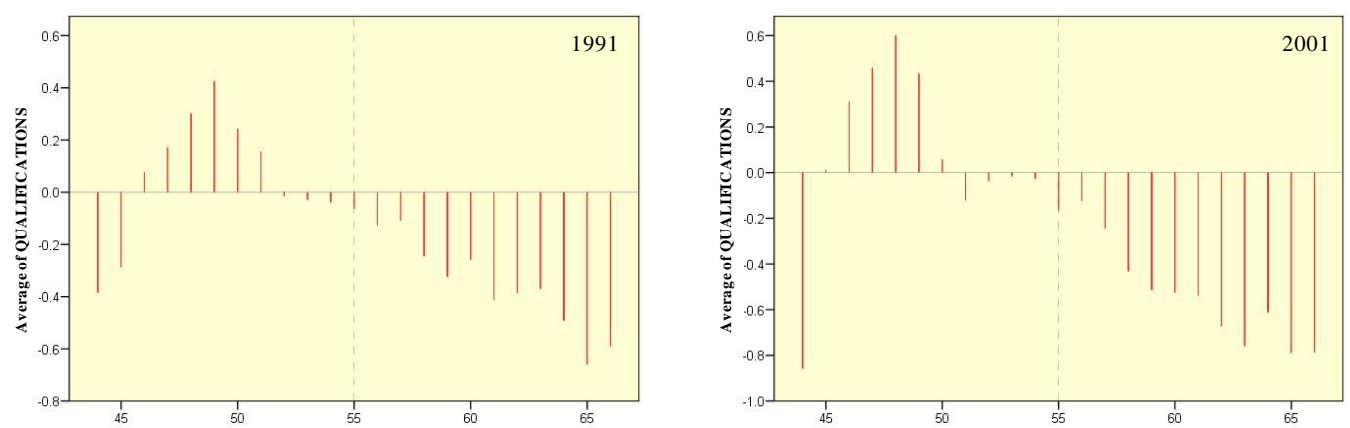

Fig.3: Pedestrian noise exposures and socio-economic factor scores

It is also relevant in terms of transport policy implications to analyse whether the disadvantage for the low-qualified employed population is cumulative to a disadvantage for the low-qualified non-employed population. The map of the areas that exceed the standards of exposures for both non-employed and employed population in 2001 [Fig.4] shows that areas with exposures above standards for both indicators and with above-average qualifications are limited to the central part of Lisbon, while the large majority of the other areas above standards have belowaverage qualifications. 


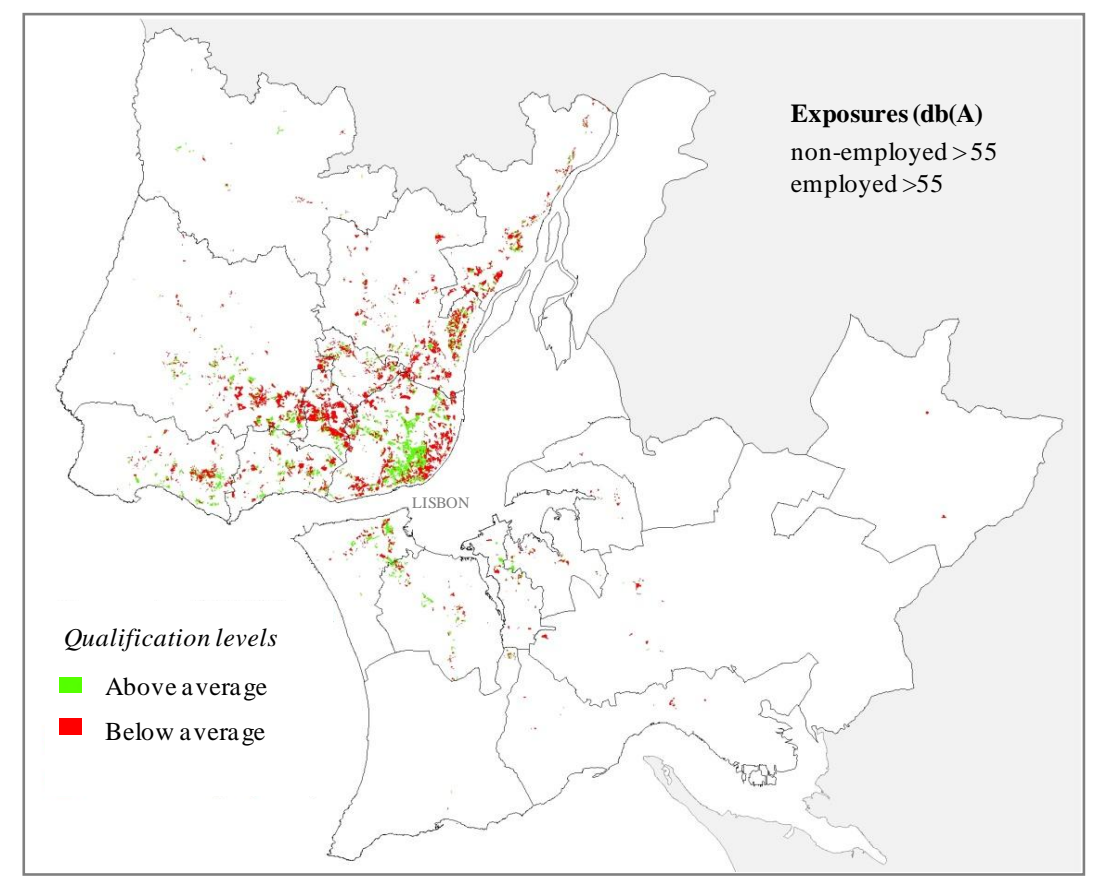

Fig. 4: Noise above standards and qualification levels

\section{Conclusions and further work}

This paper proposed a GIS-based method to measure the spatial distribution of pedestrian noise exposures in a metropolitan area. This method provides information to policy-makers that is not captured by the usual noise maps, as it integrates hypothesis about the daily mobility of the individuals affected. This information can be used in the social and environmental assessments of strategic plans in the transport sector. The application of our method to the case of the Lisbon Metropolitan Area showed that there is a variety of different spatial patterns in the distribution of exposures, which can apply in other urban areas. These patterns are based on the location of large transport infrastructure, commuting corridors and different areas within each municipality. The analysis also provides insights on equity aspects related to transport planning. The expansion of the road infrastructure and traffic in the study area contributed to increased exposures for the non-employed individuals in areas with predominantly elderly populations, while not reducing the differences in exposures for the employed individuals of different qualification groups.

The research also raises questions for further analysis regarding the role of noise as a factor restricting pedestrian mobility. Subjective assessments of noise annoyance do not depend only on noise levels but also on other sound attributes (such as the duration and frequency of noise events), the characteristics of the individual exposed, and the physical and social environment. The analysis in this paper can then be extended to include further measures of noise (such as quantile-based measures) and information on people's perceptions about the sound attributes of different noise sources in different parts of the city at different times. 


\section{Acknowledgements}

This research was supported by the Portuguese Foundation for Science and Technology. Thanks go to Steve Gibbons, Helena Titheridge and Andrew Lovett for their comments and suggestions.

\section{References}

1. Korte, C., Grant, R. (1980) Traffic noise, environmental awareness, and pedestrian behavior. Environment and Behaviour 12, 408-420.

2. Appleyard, D. (1981) Livable Streets. University of California Press, Berkeley.

3. Manaugh, K., El-Geneidy, A. (2011) Validating walkability indices: How do different households respond to the walkability of their neighborhood? Transportation Research D 16, 309-315.

4. Witten, K., Pearce, J., Day, P. (2011) Neighbourhood Destination Accessibility Index: a GIS tool for measuring infrastructure support for neighbourhood physical activity. Environment and Planning A 43, 205-223.

5. Marshall, J D., Brauer, M., Frank, L D. (2009) Healthy neighborhoods: Walkability and air pollution. Environmental Health Perspectives 117, 1752-1759.

6. Brainard, J., Jones, A., Bateman, I., Lovett, A. (2003) Exposure to environmental urban noise pollution in Birmingham, UK. Urban Studies 41, 2581-2600. 